\title{
From spectral broadening to recompression: dynamics of incoherent optical waves propagating in the fiber
}

\author{
Jun Ye, Xiaoya Ma, Yang Zhang, Jiangming Xu*, Hanwei Zhang, Tianfu Yao, Jinyong Leng and Pu Zhou*
}

\author{
* Correspondence: jmxu1988@163. \\ com; zhoupu203@163.com \\ College of Advanced \\ Interdisciplinary Studies, National \\ University of Defense Technology, \\ 410073 Changsha, China
}

\begin{abstract}
Interplay between dispersion and nonlinearity in optical fibers is a fundamental research topic of nonlinear fiber optics. Here we numerically and experimentally investigate an incoherent continuous-wave (CW) optical field propagating in the fiber with normal dispersion, and introduce a distinctive spectral evolution that differs from the previous reports with coherent mode-locked fiber lasers and partially coherent Raman fiber lasers [Nat. Photonics 9, 608 (2015).]. We further reveal that the underlying physical mechanism is attributed to a novel interplay between groupvelocity dispersion (GVD), self-phase modulation (SPM) and inverse four-wave mixing (IFWM), in which SPM and GVD are responsible for the first spectral broadening, while the following spectral recompression is due to the GVD-assisted IFWM, and the eventual stationary spectrum is owing to the dominant contribution of GVD effect. We believe this work can not only expand the light propagation in the fiber to a more general case and help advance the physical understanding of light propagation with different statistical properties, but also benefit the applications in sensing, telecommunications and fiber lasers.
\end{abstract}

Keywords: Nonlinear fiber optics, incoherent light, propagation dynamics, spectral recompression

\section{Introduction}

Dispersion and nonlinearity play important roles in fiber-based devices and systems. For example, dispersion-induced pulse broadening limits the capacity of fiber-optic communication systems [1]. Nonlinear Kerr effects such as self-phase modulation (SPM) and four-wave mixing (FWM) may contribute to the spectral broadening of optical waves [2], which is undesirable for some applications, e.g., narrow-linewidth fiber lasers [3], but benefits the novel light sources such as supercontinuum generation [4]. In general, the optical waves propagating in the fiber are affected by dispersion and nonlinear effects simultaneously, and the interplay between dispersion and different nonlinear effects leads to a multitude of interesting phenomena in nonlinear fiber optics, such as the conservative optical solitons which are enabled by the exact balance between dispersion and Kerr nonlinearity [5], and supercontinuum generation whose

(c) The Author(s). 2021 Open Access This article is licensed under a Creative Commons Attribution 4.0 International License, which permits use, sharing, adaptation, distribution and reproduction in any medium or format, as long as you give appropriate credit to the original author(s) and the source, provide a link to the Creative Commons licence, and indicate if changes were made. The images or other third party material in this article are included in the article's Creative Commons licence, unless indicated otherwise in a credit line to the material. If material is not included in the article's Creative Commons licence and your intended use is not permitted by statutory regulation or exceeds the permitted use, you will need to obtain permission directly from the copyright holder. To view a copy of this licence, visit http://creativecommons.org/licenses/by/4.0/. 
spectral evolution is affected not only by a variety of nonlinear effects but also by the dispersive properties of the fiber [6]. Several decades of investigations on nonlinear fiber optics have undoubtedly, not only promoted the growth of fundamental nonlinear physics $[7,8]$, but also benefited the engineering of fiber-based devices and systems $[9,10]$.

The propagation of optical waves in the fiber has become a simple but effective way for the investigations of interplay between dispersion and nonlinear effects [11-14]. The most extensively studied case is the propagation of ultrashort pulses. The interplay between dispersion, nonlinearity and other effects (such as mode coupling and dissipative effect) has resulted in a variety of optical solitons [15-18] and extreme-amplitude rogue waves $[19,20]$. Focusing on the spectral evolutions, the interplay between groupvelocity dispersion (GVD) and SPM effects leads to a monotonic but gradually saturated spectral broadening in the normal dispersion regime [2, 21], while soliton fission, dispersive wave, Raman solitons, and nonlinear interactions among them are responsible for the supercontinuum generation in the anomalous dispersion regime [22]. The partially coherent continuous-wave $(\mathrm{CW})$ laser beams propagating in the fiber also attracted considerable attention in recent years [23-31]. A series of distinctive spectral evolutions such as symmetric spectral broadening [23, 24], asymmetric spectral broadening [25], damped oscillations of the spectral tails [26], and supercontinuum generation are reported [27]. And besides the nonlinear Schrödinger equation (NLSE) [28], kinds of theories including the fully stochastic model [29], the phenomenological model [30], and the wave kinetics approach are established to describe the light propagation [31]. In 2015, S. K. Turitsyn et al. investigated the partially coherent Raman fiber lasers propagating in the fiber, and demonstrated a new nonlinear effect called inverse four-wave mixing (IFWM) [32], which may occur in the normal dispersion regime and cause the spectral compression of incident light. It's an exciting progress but remains an open question as how to expand the light propagation in the fiber to a more general case. As it was known that, the statistical properties of incident waves play an important role in the spectral evolutions [33]. In fact, the propagation of coherent waves (e.g., mode-locked lasers) has been studied extensively for decades, and the propagation of partially coherent waves such as Raman lasers has also been investigated in recent years. However, the incoherent waves (such as thermal light sources and free-running lasers) propagating in the fiber and the underlying dynamics, which are both scientifically important and of great interest, have been relatively unexplored.

In this article, we numerically and experimentally investigated the spectral properties of incoherent $\mathrm{CW}$ optical waves propagating in the fiber with normal dispersion, and introduced a distinctive spectral evolution that differs from the previous reports with coherent mode-locked fiber lasers and partially coherent Raman fiber lasers. The numerical simulations revealed that the underlying physical mechanism was attributed to a novel interplay between GVD, SPM and IFWM effects. The spectral recompression is particularly found to be the result of the GVD-assisted IFWM effect. And propagation experiments employing an amplified spontaneous emission (ASE) source as the input were carried out, which agreed well with the numerical simulations. We believe this work can help expand the light propagation in the fiber to a more general case, broaden the physical understanding of light propagation with different statistical properties, and benefit the applications in sensing, telecommunications and fiber lasers. 


\section{Results}

\section{Numerical simulation of spectral evolutions}

To investigate the influence of initial spectral width on the spectral evolutions of an incoherent CW optical field propagating in the fiber, we first carried out the simulations with a relatively narrow input spectrum $(0.1 \mathrm{~nm}$ full-width at half maximum (FWHM) linewidth) and a relatively broad input spectrum (0.5 nm FWHM linewidth), and the spectral profile is assumed to be Gaussian for the sake of simplicity (see 'Methods' section for details). A similar trend of spectral evolutions is confirmed in the simulations, where first spectral broadenings followed by recompressions are observed (although the broad input spectrum shows no major changes). The input spectra, the broadest spectra and the eventual spectra after a rather long propagation distance (35 $\mathrm{km}$ ) are shown in Fig. 1A, in which the input power $P_{0}$ is fixed at $1.2 \mathrm{~W}$. The spectrum with $0.1 \mathrm{~nm}$ initial FWHM linewidth shows a considerable broadening over $\sim 2 \mathrm{~km}$ propagation distance, after that a recompression of the central part takes place while the tails are nearly unchanged. The spectral evolution with $0.5 \mathrm{~nm}$ initial FWHM linewidth undergoes a similar but less pronounced process, and the recompression point (the propagation distance where the spectral recompression occurs) moves backwards to $\sim 350 \mathrm{~m}$.

The spectral evolution shows a convergence after a quite long propagation distance. Interestingly, the eventual stable spectrum is neither fully Gaussian nor fully Lorentzian, and the corresponding spectra plotted in a logarithmic scale further verified this characteristic (see Fig. 1B). Indeed, the output spectrum looks more like a sum of two Gaussians, thus it cannot be fully expressed by a single parameter [23]. Here we introduce both FWHM and root-mean-square (RMS) to describe the spectral width, and the corresponding spectral broadening factors (BFs) defined as $\Delta \lambda_{\text {out }} / \Delta \lambda_{\text {in }}$ are plotted in

(a)

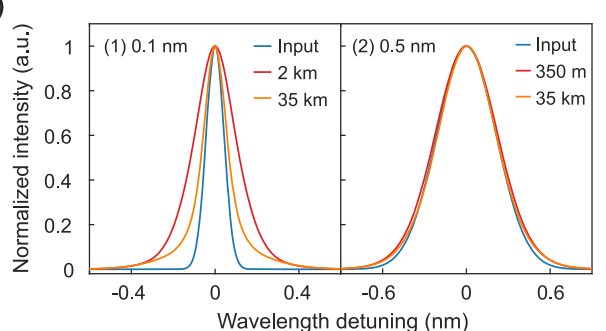

(b)

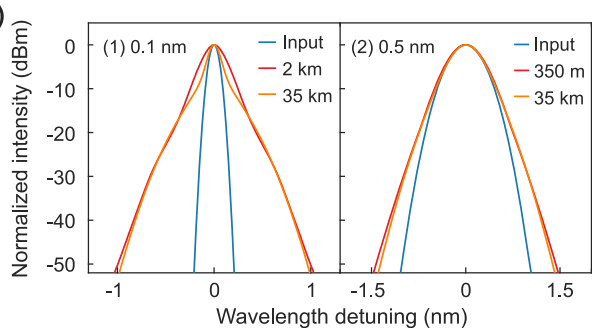

(c)

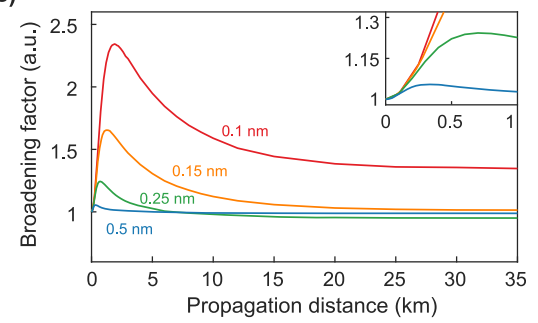

(d)

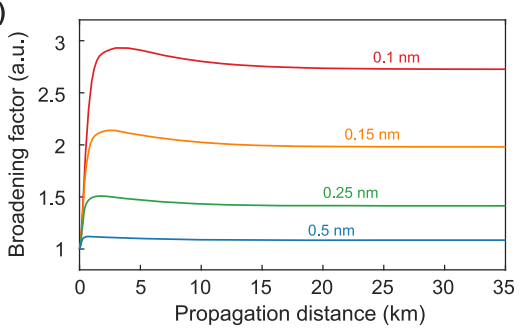

Fig. 1 Simulated spectral evolutions. A Simulated optical spectra in a linear scale. B Simulated optical spectra in a logarithmic scale. (a-1) and (b-1): $0.1 \mathrm{~nm}$ initial FWHM linewidth. (a-2) and (b-2): $0.5 \mathrm{~nm}$ initial FWHM linewidth. The input spectra, the broadest spectra and the eventual stable spectra are shown as blue, red and orange lines, respectively. C, D Spectral broadening factors dependence on the propagation distance, calculated using (C) FWHM linewidth and (D) RMS linewidth. The inset of (C) shows a zoomed-in plot within the propagation distance of $1 \mathrm{~km}$. Note that the input power $P_{0}$ is fixed at $1.2 \mathrm{~W}$ in all the simulations 
Fig. 1C, D [30, 32]. The FWHM-BF shows a rapid increase in the early stage of propagation, after that the spectral recompression occurs, the FWHM-BF gradually decreases and eventually tends to a stable value as the propagation distance increases. The maximum FWHM-BF decreases dramatically, and the recompression also takes place at a shorter propagation distance as the initial spectral FWHM increases. For example, the maximum FWHM-BF with $0.1 \mathrm{~nm}$ initial FWHM linewidth reaches $\sim 2.34$ at the propagation distance of $\sim 2 \mathrm{~km}$, while that with $0.5 \mathrm{~nm}$ initial FWHM linewidth decreases to $\sim 1.05$, and the recompression point also moves backwards to $\sim 350 \mathrm{~m}$. Further simulations find that with a broader input spectrum, the maximum FWHM-BF will tend towards 1 , and the recompression point will move to 0 , indicating that the spectrum will keep almost unchanged as the optical field propagates down the fiber (details are provided in Supplementary Section S1). In addition, an interesting feature is observed with 0.25 and $0.5 \mathrm{~nm}$ initial FWHM linewidth, where the eventual FWHM-BF decreases to $\sim 0.95$ and $\sim 0.99$, respectively, indicating that the eventual output spectra become narrower than the input ones (although the narrowing seems not so pronounced). The RMS-BF shows a similar evolution, with the only difference that the decrease looks less evident (Fig. 1D), which implies that the spectral tails are nearly unchanged during the recompression stage. Besides, in contrast to FWHM-BFs, the eventual spectral BFs calculated with RMS linewidth will not decrease to $<1$.

\section{Dynamics of spectral evolution}

To reveal the underlying physical mechanism of the distinctive spectral evolution, we focus on a snapshot of the optical field and analyze its evolution in both spectral and temporal domains. Without loss of generality, the case with $0.1 \mathrm{~nm}$ initial FWHM linewidth is chosen as an example. At the input, the temporal intensity profile within a time range of 200 ps mainly consists of two intense 'unchirped pulses' (see Fig. 2A, a broader view is shown in Supplementary Fig. S2) [34]. After a propagation distance of $0.6 \mathrm{~km}$, the optical spectrum broadens due to the SPM effect, which generates red-shifted frequency components at the leading edges and blue-shifted frequency components at the trailing edges of the pulses [2]. At the same time, the GVD effect makes the original lowfrequency and newly generated red-shifted frequency components move to the leading edges, and the original high-frequency and newly generated blue-shifted components move to the trailing edges, resulting in the broadened and chirped pulses (see Fig. 2B).

When the optical field further propagates to $1.2 \mathrm{~km}$, owing to the combined effects of SPM and GVD, the blue-shifted frequency components near the trailing edge of the preceding pulse and the red-shifted frequency components near the leading edge of the following pulse overlap in the temporal domain [35], the so-called IFWM effect takes place and regenerates the central-frequency components [32], resulting in a bridge-like area in the spectrogram and a new pulse in the temporal profile (see Fig. 2C). With more and more central-frequency components being regenerated, the optical spectrum starts to recompress, and the newly generated pulse becomes more and more intense (see Fig. 2D). As the propagation distance further increases, the whole process described above will repeat itself. Eventually, the temporal profile evolves into a bunch of random pulses with much narrower durations. And due to the fiber attenuation, the pulse intensities decrease exponentially so that the nonlinear effects become weaker 

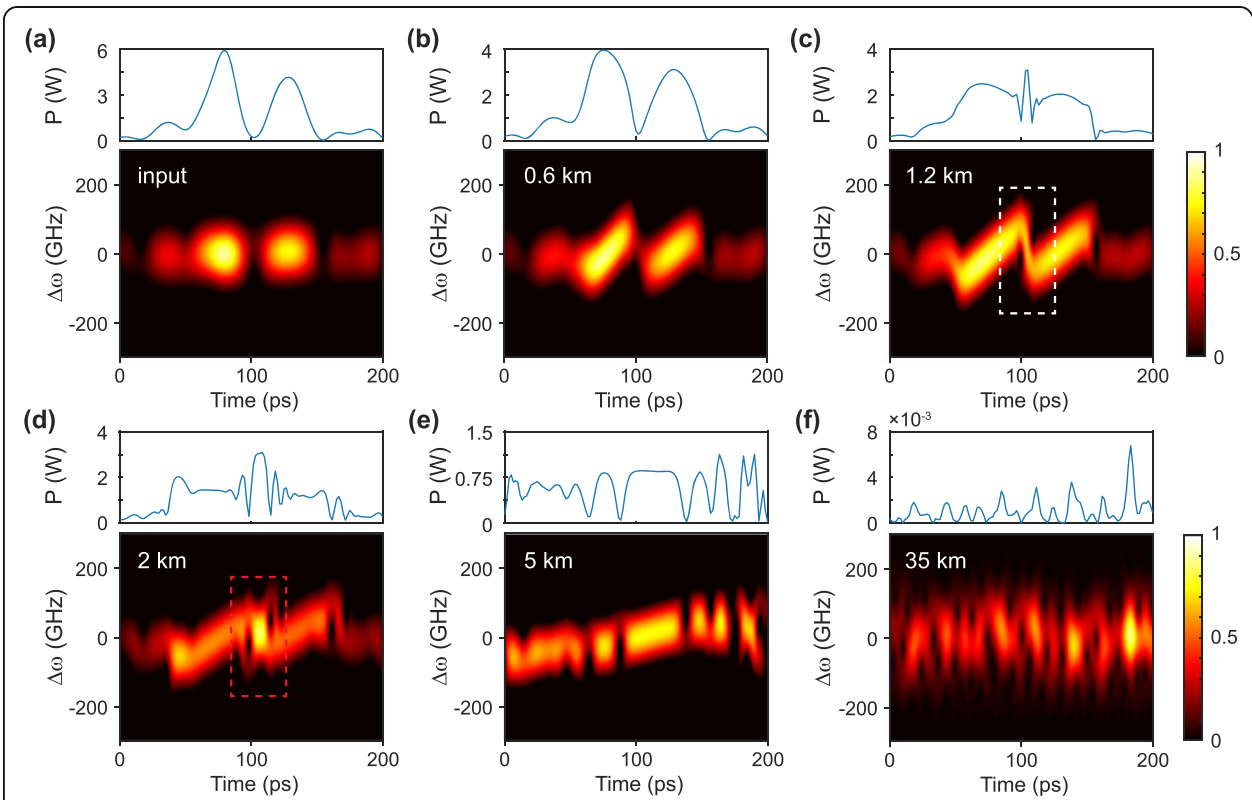

Fig. 2 Dynamics of an incoherent wave propagating in the normal dispersion regime of optical fiber. Temporal intensity profiles and spectrograms of the optical field at different propagation distances (with $0.1 \mathrm{~nm}$ initial FWHM linewidth and $1.2 \mathrm{~W}$ input power): A Input. B $0.6 \mathrm{~km}$. C $1.2 \mathrm{~km}$. D $2 \mathrm{~km}$. E $5 \mathrm{~km}$. F $35 \mathrm{~km}$. The upper inset of each picture shows the temporal intensity profile and the lower one depicts the corresponding spectrogram. The white dashed rectangular in (C) illustrates the GVDassisted IFWM, which forms a bridge-like area in the spectrogram. The red dashed rectangular in (D) highlights the regenerated central-frequency components

and weaker (see Fig. 2E, F). After a rather long propagation distance, the GVD effect dominates the field evolution, and the adjacent pulses exchange their energies frequently, resulting in a stationary optical spectrum and a kinetic equilibrium of the spectrogram (see Supplementary Video S1 for the whole dynamic process). Besides the evolution dynamics of a snapshot, we also investigated the evolutions of statistical properties as the incoherent optical field propagates along the fiber, the results show that the spectral components are uncorrelated at the input, noticeably correlated in the middle distance, and uncorrelated again after a rather long propagation distance (see Supplementary Section S3 for details).

The physical mechanism described above also explains the evolution differences with broader input spectra. Since it was known that, a broader initial spectral width corresponds to a narrow fluctuation duration (see 'Methods' section) [34], thus the blue-shifted frequency components of the preceding pulse and the redshifted frequency components of the following pulse will overlap at a shorter propagation distance, the IFWM effect and spectral recompression will also occur earlier. Correspondingly, the accumulation of the SPM effect as well as the maximum spectral BF decreases with the increase of initial spectral width. The evolution dynamics and statistical properties with initial FWHM linewidth of $0.5 \mathrm{~nm}$ are simulated and presented in Supplementary Section S4 and Supplementary Section S5, respectively.

According to the physical mechanism described above, we have derived an approximate equation governing the recompression point $L_{r}$ (The detailed derivation is provided in Supplementary Section S6) [2]: 


$$
L_{r} \Delta \omega_{0}\left|\beta_{1}\left(\omega_{0}\right)-\beta_{1}\left(\omega_{0}+\delta \omega_{\max }\right)\right|-1=0,
$$

where $\beta_{1}$ represents the first-order dispersion coefficient, $\Delta \omega_{0}$ is the $1 /$ e half-width of the optical spectrum, $\delta \omega_{\max }$ stands for the maximum SPM-induced frequency chirp which satisfies:

$$
\delta \omega_{\max }=\sqrt{2} \exp \left(-\frac{1}{2}\right) \gamma \Delta \omega_{0} P_{0}^{2}\left[1-\exp \left(-\alpha L_{r}\right)\right] / \alpha,
$$

where $P_{0}$ is the average input power, $\alpha$ and $\gamma$ stand for the attenuation coefficient and Kerr nonlinearity coefficient, respectively. By substituting Eq. (2) into Eq. (1), the solution of recompression point $L_{r}$ can be obtained. Then the maximum RMS-BF $B F_{\max }$ is given by [36]:

$$
B F_{\max }=\sqrt{1+\frac{4}{3 \sqrt{3}} \frac{\gamma^{2} P_{0}^{2}\left[1-\exp \left(-\alpha L_{r}\right)\right]^{2}}{\alpha^{2}}} .
$$

Figure $3 \mathrm{~A}, \mathrm{~B}$ show the dependence of recompression point and maximum RMS-BF on the initial FWHM linewidth. As can be seen, the solutions of Eq. (1) and Eq. (3) agree well with the NLSE-based simulations. As the initial spectral FWHM increases from 0.1 to $2 \mathrm{~nm}$, the recompression point moves from $\sim 1750 \mathrm{~m}$ backwards to $\sim 88 \mathrm{~m}$, and the maximum RMS-BF decreases dramatically from $\sim 2.93$ to $\sim 1.01$.

\section{Experimental setup}

ASE source which originates from the random thermal noise, is assumed to be a good approximation of incoherent CW optical field [34, 37]. With the aid of a linewidthtunable 1064 nm ASE source [38] and sections of G.652.D fibers, we experimentally studied the spectral evolutions of ASE light propagating in the normal dispersion regime of optical fiber. The experimental setup is illustrated in Fig. 4, since the minimal FWHM linewidth of the ASE source is $\sim 0.4 \mathrm{~nm}$ and to obtain a narrower spectrum, the ASE source is spliced with a circulator, followed by a highly reflective $1064 \mathrm{~nm}$ fiber Bragg grating (FBG). Thus, a narrower optical spectrum (orange line) reflected by the FBG can be obtained in port-3 of the circulator, while the transmitted spectrum is converted to a double-peak profile (blue line). Sections of G.652.D fibers with a length ranging from $100 \mathrm{~m}$ to $35 \mathrm{~km}$ are used as the transmission media, and an optical spectrum analyzer (OSA) with a resolution of $0.02 \mathrm{~nm}$ is used to measure the output spectra.
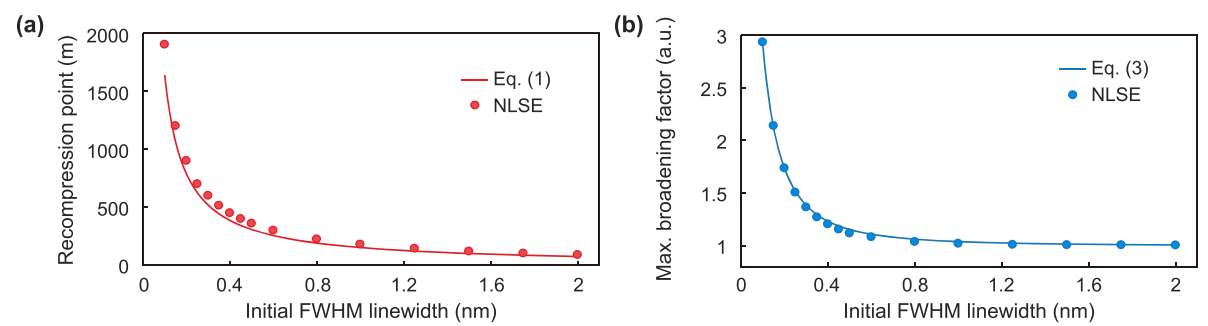

Fig. 3 Comparison between the results of NLSE and approximate equations. A Recompression point as a function of initial FWHM linewidth. B Maximum RMS-BF dependence on the initial FWHM linewidth. Lines: solutions of the approximate equations. Scatters: simulated results based on NLSE 


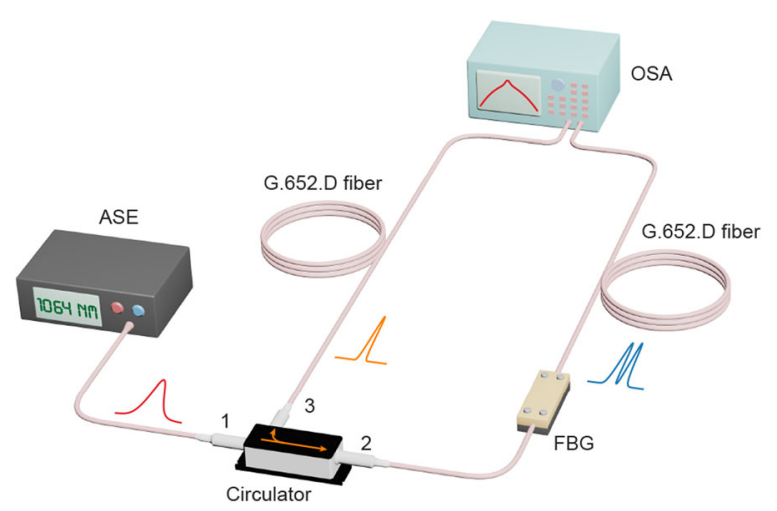

Fig. 4 Schematic of the experimental setup. ASE, amplified spontaneous emission; FBG, fiber Bragg grating; OSA, optical spectrum analyzer

\section{Spectral evolution with single-peak spectrum}

We first carried out the propagation experiments of the single-peak spectrum. Three cases with different spectral widths are investigated, two of them are realized by the reflection of FBGs (0.11 and $0.23 \mathrm{~nm}$ reflection bandwidth, respectively), and the last one is obtained by directly removing the FBG and adjusting the FWHM linewidth of the ASE source to $1.47 \mathrm{~nm}$ (recalling that the ASE source is linewidth-tunable with a minimal FWHM linewidth of $\sim 0.4 \mathrm{~nm}$ ). Figure $5 \mathrm{~A}-\mathrm{C}$ depict the spectral evolutions in a linear scale with the initial FWHM linewidth of $0.11,0.23$ and $1.47 \mathrm{~nm}$, respectively. The input spectrum with $0.11 \mathrm{~nm}$ FWHM linewidth shows a Gaussian-like profile, while that with $0.23 \mathrm{~nm}$ FWHM linewidth looks a little irregular, and the spectrum with $1.47 \mathrm{~nm}$ FWHM linewidth approaches a super-Gaussian shape. As can be seen, the optical spectrum with $0.11 \mathrm{~nm}$ FWHM linewidth varies significantly during the propagation, showing a dramatic spectral broadening in the near $2 \mathrm{~km}$ and a noticeable recompression in the latter propagation distance. A similar evolution is observed with $0.23 \mathrm{~nm}$ initial FWHM linewidth. However, in contrast to the two cases above, the optical spectrum with $1.47 \mathrm{~nm}$ initial FWHM linewidth remains stationary during the whole propagation process.

Interestingly, more details can be found in the spectral wings (see Fig. 5D-F for the optical spectra in a logarithmic scale). Indeed, the input spectrum with 0.11 and 0.23 nm FWHM linewidth is not fully Gaussian especially considering the broad tails and the irregular peaks embedded in the tails. However, the spectra become smooth, regular and symmetrical after propagation, showing a self-organization effect [39]. And the optical spectrum with $1.47 \mathrm{~nm}$ initial FWHM linewidth is not completely unchanged during the propagation, its wings instead become more and more intense as the propagation distance increases. Figure $5 \mathrm{G}$ shows the FWHM-BF as functions of the propagation distance, it should be noted that here the input power $P_{0}$ is fixed at $1.2 \mathrm{~W}$ (we also numerically and experimentally investigated the impact of input power, see Supplementary Section S7 for details). Regarding the case with $0.11 \mathrm{~nm}$ initial FWHM linewidth, the recompression occurs at $\sim 2 \mathrm{~km}$ with the maximum FWHM-BF reaching $\sim 2.14$, and the eventual FWHM-BF recompresses to $\sim 1.27$. While with $0.23 \mathrm{~nm}$ initial FWHM linewidth, the recompression point moves backwards to $\sim 1 \mathrm{~km}$ with the 


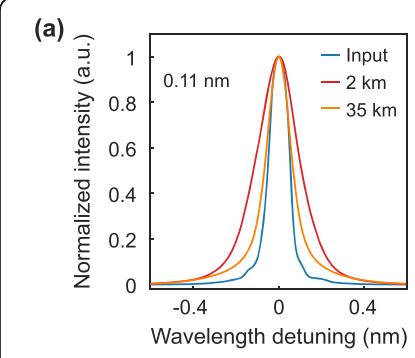

(b)

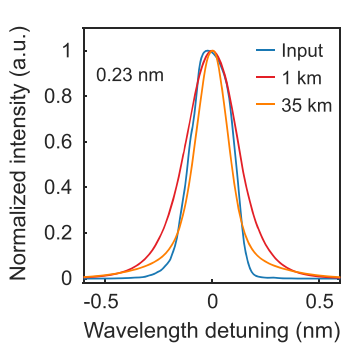

(d)

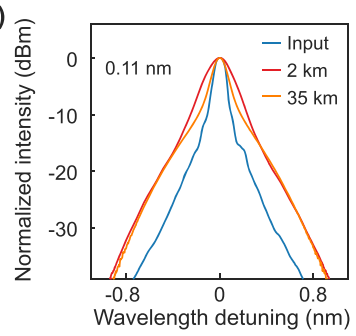

(g)

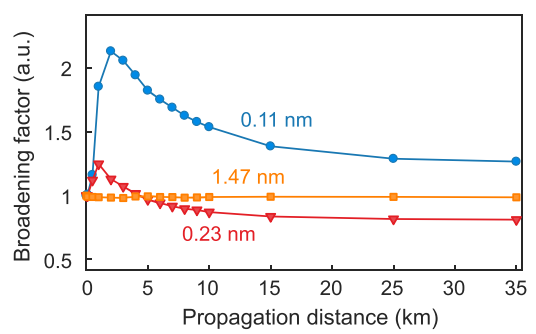

(e)

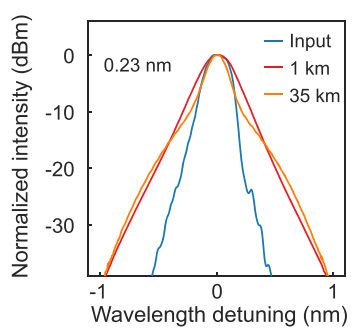

(h) (c)

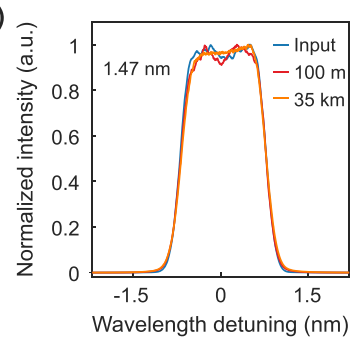

(f)

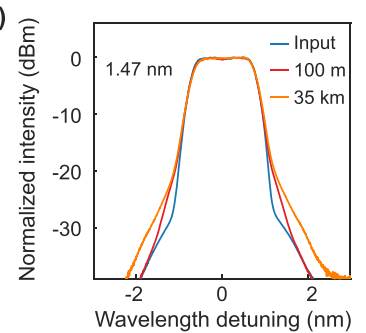

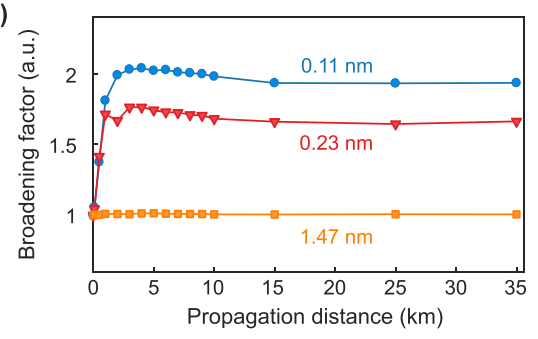

Fig. 5 Experimental spectral evolutions of single-peak spectra. Experimental optical spectra in a linear scale $(\mathbf{A}, \mathbf{B}, \mathbf{C})$ and in a logarithmic scale (D, E, F). (A) and (D): $0.11 \mathrm{~nm}$ initial FWHM linewidth. (B) and (E): 0.23 $\mathrm{nm}$ initial FWHM linewidth. (C) and (F): $1.47 \mathrm{~nm}$ initial FWHM linewidth. The input spectra, the broadest spectra and the eventual stable spectra are shown as blue, red and orange lines, respectively. G, $\mathbf{H}$ Spectral broadening factors dependence on the propagation distance, calculated using $(\mathbf{G})$ FWHM linewidth and $(\mathbf{H})$ RMS linewidth. Note that the input power $P_{0}$ is also fixed at $1.2 \mathrm{~W}$ in all the experiments

maximum FWHM-BF decreasing to $~ 1.25$, and the eventual FWHM-BF also drops to $\sim 0.81$, indicating that the output spectrum has a narrower FWHM linewidth than the input one (which has been proved to be the impact of the initial spectral profile, see Supplementary Section S8). In terms of RMS-BFs (Fig. $5 \mathrm{H}$ ), the spectral recompression seems not so pronounced, the RMS-BF with $0.11 \mathrm{~nm}$ initial FWHM linewidth slightly decreasescan also introduce the dispersion length from maximal 2.04 to eventual 1.93, and that with $0.23 \mathrm{~nm}$ initial FWHM linewidth decreases from maximal 1.76 to eventual 1.66. More interestingly, the BF with $1.47 \mathrm{~nm}$ initial spectral FWHM keeps unchanged at 1 in terms of both FWHM and RMS linewidth. Incidentally, the distinctive spectral evolutions enabled by the interplay between dispersion and nonlinearity in the propagation naturally raises an intriguing question-how the dispersion and nonlinearity parameters influence the spectral evolution, which has been numerically studied and presented in Supplementary Section S9.

The experimental spectral evolutions of incoherent $\mathrm{CW}$ optical waves propagating in the fiber with normal dispersion have well confirmed the simulation results, and their unique properties contrast sharply with those of the pulsed sources (e.g., mode-locked lasers), in which no spectral recompression occurs instead of a broadening saturation $[2,21,40]$, and they also differ from those of the Raman fiber lasers, in which a 
relatively broad initial spectrum undergoes narrowing while the propagation, and the spectral narrowing becomes more and more pronounced as the initial spectral width increases (see Fig. 6A of Ref. [32]). To summarize, here the distinctive spectral evolutions indicate not only the 'continuous' but also the 'incoherent' natures of the input optical waves.

\section{Spectral evolution with double-peak spectrum}

To further reveal the particularity of the incoherent optical field, we carried out the propagation experiment with a double-peak spectrum. The unique spectral profile is obtained with a relatively broad single-peak spectrum transmitting through the FBG, and the initial FWHM linewidth of the double-peak spectrum reaches $\sim 0.68 \mathrm{~nm}$. Figure 6A shows the normalized optical spectra in a logarithmic scale, the intensity of the central valley is $\sim 15 \mathrm{~dB}$ lower than the peaks at the input, the it increases to about $-2.5 \mathrm{~dB}$ due to the broadening and overlapping of the two peaks at $3 \mathrm{~km}$, after that a slight spectral recompression is observed, and the intensity of the central valley decreases to about $-4.2 \mathrm{~dB}$ at $35 \mathrm{~km}$. Figure $6 \mathrm{~B}$ depicts the spectral evolutions in a linear scale, as can be seen, no matter how long the optical field propagates through, the spectrum only shows a slight broadening in terms of the FWHM linewidth. Besides, the evolution trend is similar to that of the single-peak spectrum. The double-peak spectrum broadens to its maximum with $3-\mathrm{km}$-long propagation, after that a spectral recompression is observed. The intensity of the central valley also shows a similar variation, which reaches its maximum with $3-\mathrm{km}$-long propagation, then decreases as the propagation distance further increases. In contrast to the partially coherent Raman fiber lasers, the double-peak spectrum of an incoherent $\mathrm{CW}$ optical field will neither evolve to a single-peak profile nor show a spectral narrowing as propagating in the normal dispersion regime [32], it seems more like a sum of two separate single-peak spectra propagating independently, which further reveals the incoherent nature of the optical field. Figure $6 \mathrm{C}$ shows the spectral BFs as functions of the propagation distance. The FWHM-BF increases to maximal 1.13 at the propagation distance of $3 \mathrm{~km}$, then gradually recompresses to eventual 1.04, while the RMS-BF shows a maximum value of 1.08 and a convergence of 1.05 .

In addition, the random phase model of the CW incoherent optical field (see Eq. (5) of the 'Methods' section) enables us to use the experimental spectral profile with
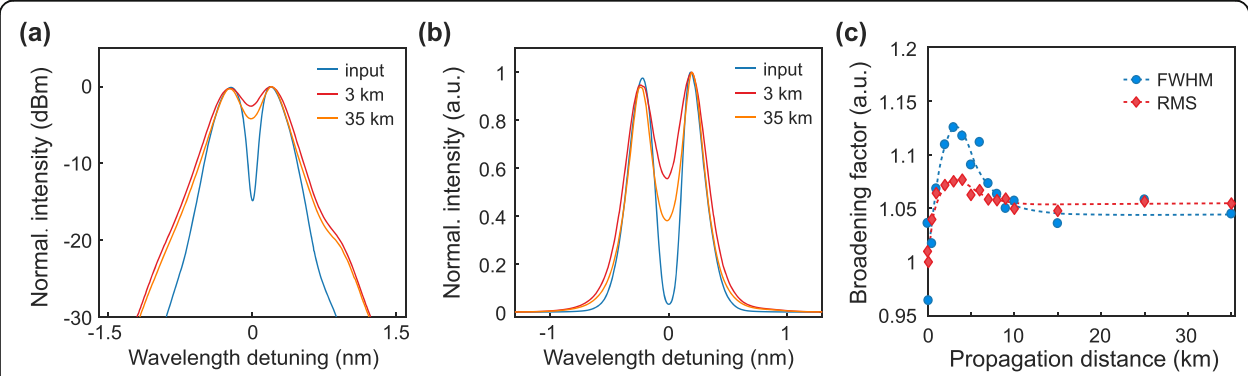

Fig. 6 Experimental spectral evolutions of double-peak spectra. A Output spectra in logarithmic scale at 3 different positions: input, 3 and $35 \mathrm{~km}$. It's worth noting that the spectral FWHM linewidth broadens to its maximum with 3-km propagation. B Output spectra in linear scale. C Spectral broadening factors dependence on the propagation distance. Scatters: experimental data; lines: spline fitting 
random phases as the input and simulated the spectral evolution of a double-peak initial spectrum. The simulation matches nicely with the experimental result (see Supplementary Section S10 for details).

\section{Discussion}

Here we would like to first discuss the evolution differences with different initial spectral widths. Regarding the dynamics, since a broader initial spectral width corresponds to a narrow fluctuation duration, the blue-shifted frequency components of the preceding pulse and the red-shifted frequency components of the following pulse will overlap at a shorter propagation distance, thus the IFWM effect and spectral recompression will also occur earlier. Correspondingly, the accumulation of the SPM effect as well as the maximum spectral BF will decrease with the increase of initial spectral width. We can also introduce the dispersion length $L_{\mathrm{D}}=T_{0}^{2} /\left|\beta_{2}\right|$ and the nonlinear length $L_{\mathrm{NL}}=1$ / $\left(\gamma P_{0}\right)$ as references. As we know, the shorter the reference length (namely, the dispersion length and nonlinear length), the stronger the corresponding effect. With the same input power, the nonlinear length is identical for different initial spectral widths. In our case, the nonlinear length is estimated to be $\sim 0.46 \mathrm{~km}$ at the input. However, the dispersion length varies with the initial spectral width (since the temporal fluctuation duration changes with the initial bandwidth). For example, the dispersion length is estimated to be $\sim 11 \mathrm{~km}$ with $0.1 \mathrm{~nm}$ initial spectral FWHM linewidth, while it decreases to $\sim 0.44 \mathrm{~km}$ with $0.5 \mathrm{~nm}$ initial spectral FWHM linewidth. Therefore, since the dispersion length is much longer than the nonlinear length $\left(L_{\mathrm{D}} / L_{\mathrm{NL}} \gg 1\right)$ for narrow initial spectral widths, the nonlinear effect (here it refers specifically to the SPM effect) will dominate the spectral evolution at the early stage, accordingly, the effect of spectral broadening is stronger for narrow initial spectral widths.

We also would like to further discuss the differences with coherent pulsed light (e.g., mode-locked fiber lasers), partially coherent CW light (e.g., Raman fiber lasers) and incoherent $\mathrm{CW}$ optical field propagating in the normal dispersion fiber. For pulsed light, the SPM effect will first broaden the optical spectrum and enhance the pulse broadening induced by the GVD effect, and the enhanced pulse broadening in turn weakens the SPM effect, eventually, the interplay between GVD and SPM effects leads to a monotonic but gradually saturated spectral broadening with the increase of propagation distance [2, 21]. For partially coherent CW optical waves such as Raman fiber lasers, the similarity with coherent mode-locked fiber lasers is that they all have a variety of longitudinal modes, the difference is the correlation degree of the multiple longitudinal modes. As we know, the longitudinal modes of a mode-locked fiber laser are phaselocked - in other words, these spectral components are coherent (or totally correlated). And the interference of these resonant modes leads to a train of ultrashort pulses. While regarding Raman fiber lasers, the turbulent FWM interaction of numerous longitudinal modes gives rise to the partial correlation of the spectral components (especially at the spectral wings), and the partial correlation results in the turbulent intensity fluctuations in the temporal domain. The spectral evolution of partially coherent Raman waves propagating in the normal dispersion fiber shows two cases: one is similar to that of incoherent optical waves propagating in the fiber - first spectral broadening followed by recompression, the other is monotonic but gradually saturated spectral narrowing [32]. In fact, the first case corresponds to a narrowband Raman fiber laser 
(usually operating at a low power), in which the turbulent FWM interaction of numerous longitudinal modes is weak, thus its output is similar to an incoherent optical wave; while the second case corresponds to a relatively broad initial spectrum (usually operating at a higher power), where the optical spectrum can be considered as an assembly of correlated spectral components generated by the FWM effect inside the cavity [41], thus the IFWM effect can occur at the beginning and compress the spectrum. Of course, SPM also takes place in this process but the IFWM effect dominates the evolution, and due to the power attenuation of light propagation, the interplay between IFWM and SPM effects leads to a monotonic but weakening spectral narrowing as the propagation distance increases (see Fig. 6D of Ref. [32]). Accordingly, we can treat the partially coherent Raman fiber laser as an intermediate state between the coherent mode-locked fiber lasers and incoherent optical waves. When the turbulent FWM interaction of numerous longitudinal modes is weak, the output field of a Raman fiber laser comes closer to an incoherent optical wave; when the turbulent FWM interaction of numerous longitudinal modes is stronger, the output field of a Raman fiber laser can be treated as the partially coherent light; in the extreme, the longitudinal modes of a Raman fiber laser are totally phase-locked (or correlated), it becomes a coherent modelocked Raman fiber laser.

In contrast to coherent mode-locked fiber lasers and partially coherent Raman fiber lasers, the optical spectrum of an incoherent $\mathrm{CW}$ field propagating in the normal dispersion regime evolves consistently no matter how broad the initial spectrum is, that is, broadens first afterwards recompresses. In the beginning, the SPM effect dominates the evolution and the IFWM effect cannot take place since the spectral components of an incoherent $\mathrm{CW}$ field are uncorrelated. Thus the initial spectral broadening is due to the interplay between SPM and GVD effects, just like the case of pulsed light. However, with more and more correlated red-shifted components and blue-shift components being generated through the SPM effect, and overlapping in the temporal domain through the GVD effect, the IFWM effect can occur and compress the optical spectrum. And since the nonlinear effects become extremely weak due to the power attenuation of long-distance propagation, the ultimate optical spectrum remains stable owing to the dominant contribution of the GVD effect. To better understand the evolution dynamics with different input optical waves, a detailed comparison is presented in Supplementary Section S11.

\section{Conclusions}

In conclusion, we numerically and experimentally investigated the propagation of an incoherent CW optical field in the fiber with normal dispersion, and demonstrated a distinctive spectral evolution that differs greatly from the previous reports with coherent mode-locked fiber lasers and partially coherent Raman fiber lasers. The results show that, no matter how broad the initial spectrum is, the output spectrum undergoes a broadening followed by a recompression as the propagation distance increases. Besides, the broader the initial spectrum, the earlier the recompression occurs, and the smaller the maximum spectral broadening factor. The underlying physical mechanism is attributed to a novel interplay between GVD, SPM and IFWM effects, in which SPM and GVD are responsible for the first spectral broadening, while the following spectral recompression is due to the GVD-assisted IFWM, and the eventual 
stationary spectrum is owing to the dominant contribution of GVD effect. According to the physical mechanism, an approximate equation governing the recompression point was derived, which agrees well with the NLSE-based simulations. Propagation experiments employing an ASE source as the input were carried out, which confirmed the results of numerical simulations. Specifically, the result with a double-peak input spectrum differs greatly from that with Raman fiber lasers, indicating the critical importance of spectral correlation on the propagation-induced spectral changes. We believe this work can not only expand the light propagation in the fiber to a more general case and help advance the physical understanding of light propagation with different statistical properties, but also benefit the applications in sensing, telecommunications and fiber lasers.

\section{Methods}

\section{Light propagation in the fiber}

The propagation of an optical field along the fiber can be modeled by the well-known NLSE [2]:

$$
\frac{\partial A}{\partial z}=-\frac{\alpha}{2} A-\frac{i \beta_{2}}{2} \frac{\partial^{2} A}{\partial T^{2}}+i \gamma|A|^{2} A,
$$

where $A(z, T)$ is the electric field envelope, $z$ is the propagation distance, $T$ represents the time in a frame of reference moving at the group velocity of the central frequency. $\alpha, \beta_{2}$ and $\gamma$ stand for attenuation coefficient, second-order dispersion coefficient and Kerr nonlinearity coefficient, respectively. The NLSE is numerically integrated using the so-called split-step Fourier method (SSFM) [2], and the following paramenters are used in the simulation: $\alpha=0.84 \mathrm{~dB} / \mathrm{km}, \beta_{2}=25 \mathrm{ps}^{2} / \mathrm{km}$ and $\gamma=1.8(\mathrm{~W} \times \mathrm{km})^{-1}$, which are the same with the fiber parameters employed in the experiment. In addition, the G.652.D fiber we used features an $8.2 \mu \mathrm{m}$ core diameter with the numerical aperture (NA) of 0.14 , supporting not only the fundamental $\mathrm{LP}_{01}$ mode but also the $\mathrm{LP}_{11}$ mode at $1064 \mathrm{~nm}$. However, we have carried out the modal decomposition to verify the single-mode propagation [42], thus proving the validity of using single-mode NLSE in the simulations (see Supplementary Section S12). What's more, the simulated spectrum via single realization is quite noisy due to the initial random phase [23], thus an ensemble average over 2400 realizations and a last filtering of the optical spectrum are conducted. Considering that each realization is independent, the simulations are performed on a parallel computing platform with 4 notes, and each note consists of 24 cores $(2.65 \mathrm{GHz}$ clock speed) and 64 GB RAM.

\section{Modeling the $\mathrm{CW}$ input field}

Before simulating the optical field propagating in the fiber, modeling the CW input field is of critical importance. However, the previously reported models such as the phase-diffusion model and CW with one photon per mode approach [25, 28], cannot properly simulate the spectral property and temporal behavior at the same time. The most promising model so far is to simulate the actual laser oscillation using the set of coupled NLSEs and rate equations (if the active gain is involved) [43, 44]. However, for precisely simulating the spectral shape and the phase relationship between different spectral components, not only the experimental mirror profiles but also the exact 
values of fiber parameters are required $[45,46]$, which makes this approach quite complex and challenging. Fortunately, the case would be much simpler if assuming the incoherent optical field is a random complex field, then the input field can be expressed as a sum of Fourier components [47]:

$$
A(z=0, t)=\sum_{m} \hat{X}_{m} \exp (i m \Delta \omega t)
$$

where the Fourier modes $\hat{X}_{m}=\left|\hat{X}_{m}\right| \exp \left(i \phi_{m}\right)$ are complex variables with random phases $\phi_{m}$ uniformly distributed between 0 and $2 \pi[48,49]$. Accordingly, the power spectrum $A(z=0, t)$ of the random field reads:

$$
\left\langle\hat{X}_{m} \hat{X}_{n}\right\rangle=n_{0 m} \delta_{m n}=n_{0}\left(\omega_{m}\right) .
$$

And for the sake of simplicity, the initial optical power spectrum is assumed to be Gaussian with the FWHM linewidth of $\Omega_{L}$ :

$$
n_{0}(\omega)=n_{0} \exp \left[-4 \ln (2) \frac{\omega^{2}}{\Omega_{L}^{2}}\right] .
$$

Supplementary Fig. S13A shows the simulated temporal intensities $|A(z=0, t)|^{2}$ for four spectral FWHM linewidths as indicated. It's clear that the temporal intensity profile of the so-called CW optical field is not constant, in contrast, it's comprised of a random succession of fluctuations with time scales of picoseconds, and the fluctuation duration decreases as the spectral width increases [37]. The intensity autocorrelation functions (ACFs) illustrated in Supplementary Fig. S13B show the average durations of the temporal fluctuations, indicating a decrease from $\sim 16.2$ to $\sim 3.3$ ps with the spectral FWHM linewidth increasing from 0.1 to $0.5 \mathrm{~nm}$. Obviously, an incoherent CW optical field with such intense temporal fluctuations propagating in the fiber will undergo a variety of nonlinear effects, e.g., the SPM effect, which will in turn reshape the temporal intensities as well as the spectral properties of the optical field.

\section{Experimental setup of the ASE source}

The ASE source is counter-pumped by a $976 \mathrm{~nm}$ laser diode through a $(2+1) \times 1$ signal-pump combiner, the pump light is launched into 8-m-long 10/130 $\mu \mathrm{m} \mathrm{Yb-doped}$ fiber with a cladding absorption of $\sim 4 \mathrm{~dB} / \mathrm{m}$. To eliminate the end feedback induced parasitic lasing, two broadband isolators are utilized to improve the isolation ratio. The broadband ASE source is spliced with a bandwidth-tunable optical filter, through which a linewidth tuning range of $0.4-15 \mathrm{~nm}$ can be obtained.

\section{Abbreviations}

CW: continuous-wave; GVD: group-velocity dispersion; SPM: self-phase modulation; IFWM: inverse four-wave mixing; FWM: four-wave mixing; NLSE: nonlinear Schrödinger equation; ASE: amplified spontaneous emission; FWHM: fullwidth at half maximum; RMS: root-mean-square; BF: broadening factor; FBG: fiber Bragg grating; OSA: optical spectrum analyzer; SSFM: split-step Fourier method; NA: numerical aperture; ACF: autocorrelation function

\section{Supplementary information}

The online version contains supplementary material available at https://doi.org/10.1186/s43074-021-00037-x.

Additional file 1.

Additional file 2.

Additional file 3. 


\section{Acknowledgements}

The authors thank Jiaxin Song and Dr. Zhiyuan Dou for help with the spectral measurement, Yi An and Dr. Liangjin Huang for assistance with the modal decomposition, and Dr. Jian Wu for providing the computing resources. We also acknowledge Prof. Zhichao Luo and Prof. Wencheng Xu from South China Normal University for fruitful discussions.

\section{Authors' contributions}

All the authors contributed to the interpretation of the results and manuscript writing. J.Y., X.M., and Y.Z. performed the experiments; H.Z., J.X., and P.Z. conceived the idea; J.Y. conducted the numerical simulation; T.Y. and J.L. provided the technical support in experiment and data analysis; J.X. and P.Z supervised the project. The author(s) read and approved the final manuscript.

\section{Funding}

National Natural Science Foundation of China (NSFC) (61905284, 62035015, 62061136013).

\section{Availability of data and materials}

The datasets used and/or analysed during the current study are available from the corresponding author on reasonable request.

\section{Declarations}

\section{Competing interests}

The authors declare that they have no competing interests.

Received: 27 May 2021 Accepted: 15 July 2021

Published online: 04 August 2021

\section{References}

1. Ellis AD, McCarthy ME, Al Khateeb MAZ, Sorokina M, Doran NJ. Performance limits in optical communications due to fiber nonlinearity. Adv Opt Photonics. 2017;9:429-503.

2. Agrawal GP. Nonlinear fiber optics. Fifth edition. Amsterdam: Elsevier; 2013.

3. Song J, Ma P, Ren S, Zhang S, Liu W, Xiao H, et al. 2 kW narrow-linewidth Yb-Raman fiber amplifier. Opt Lett. 2021;46: 2404-7.

4. Jiang X, Joly NY, Finger MA, Babic F, Wong GKL, Travers JC, et al. Deep-ultraviolet to mid-infrared supercontinuum generated in solid-core ZBLAN photonic crystal fibre. Nat Photonics. 2015;9:133-9.

5. Song Y, Shi X, Wu C, Tang D, Zhang H. Recent progress of study on optical solitons in fiber lasers. Appl Phys Rev. 2019; 6:21313.

6. Dudley JM, Genty G, Coen S. Supercontinuum generation in photonic crystal fiber. Rev Mod Phys. 2006;78:1135-84.

7. Randoux S, Gustave F, Suret P, El G. Optical random Riemann waves in integrable turbulence. Phys Rev Lett. 2017;118: 233901.

8. Dudley JM, Taylor JR. Ten years of nonlinear optics in photonic crystal fibre. Nat Photonics. 2009;3:85-90.

9. Bao X, Chen L. Recent progress in distributed fiber optic sensors. Sensors. 2012;12:8601-39.

10. Turitsyn SK, Bale BG, Fedoruk MP. Dispersion-managed solitons in fibre systems and lasers. Phys Rep. 2012;521:135-203.

11. Reeves WH, Skryabin DV, Biancalana F, Knight JC, Russell PS, Omenetto FG, et al. Transformation and control of ultrashort pulses in dispersion-engineered photonic crystal fibres. Nature. 2003;424:511-5.

12. Fermann ME, Kruglov VI, Thomsen BC, Dudley JM, Harvey JD. Self-similar propagation and amplification of parabolic pulses in optical fibers. Phys Rev Lett. 2000;84:6010-3.

13. Armaroli A, Conti C, Biancalana F. Rogue solitons in optical fibers: a dynamical process in a complex energy landscape? Optica. 2015;2:497-504

14. Klas R, Kirsche A, Gebhardt M, Buldt J, Stark H, Hädrich S, et al. Ultra-short-pulse high-average-power megahertzrepetition-rate coherent extreme-ultraviolet light source. PhotoniX. 2021:2:4

15. Liu M, Wei ZW, Li H, Li TJ, Luo AP, Xu WC, et al. Visualizing the "invisible" soliton pulsation in an ultrafast laser. Laser Photonics Rev. 2020;14:1900317.

16. Zhang H, Tang DY, Zhao LM, Xiang N. Coherent energy exchange between components of a vector soliton in fiber lasers. Opt Express. 2008;16:12618-23.

17. Renninger WH, Wise FW. Optical solitons in graded-index multimode fibres. Nat Commun. 2013:4:1719.

18. Peng J, Boscolo S, Zhao Z, Zeng H. Breathing dissipative solitons in mode-locked fiber lasers. Sci Adv. 2019;5:eaax1110.

19. Xu J, Wu J, Ye J, Song J, Yao B, Zhang H, et al. Optical rogue wave in random fiber laser. Photonics Res. 2020;8:1-7.

20. Song $Y$, Wang Z, Wang C, Panajotov K, Zhang H. Recent progress on optical rogue waves in fiber lasers: status, challenges, and perspectives. Adv photonics. 2020:2:24001.

21. Weidner P, Penzkofer A. Spectral broadening of picosecond laser pulses in optical fibres. Opt Quant Electron. 1993:25:1-25.

22. Eftekhar MA, Sanjabi-Eznaveh Z, Lopez-Aviles HE, Benis S, Antonio-Lopez JE, Kolesik M, et al. Accelerated nonlinear interactions in graded-index multimode fibers. Nat Commun. 2019;10:1638.

23. Barviau B, Randoux S, Suret P. Spectral broadening of a multimode continuous-wave optical field propagating in the normal dispersion regime of a fiber. Opt Lett. 2006;31:1696.

24. Liu W, Ma P, Zhou P, Jiang Z. Spectral property optimization for a narrow-band-filtered superfluorescent fiber source. Laser Phys Lett. 2018;15:25103.

25. Mussot A, Lantz E, Maillotte H, Sylvestre T, Finot C, Pitois S. Spectral broadening of a partially coherent CW laser beam in single-mode optical fibers. Opt Express. 2004;12:2838-43.

26. Suret P, Picozzi A, Randoux S. Wave turbulence in integrable systems: nonlinear propagation of incoherent optical waves in single-mode fibers. Opt Express. 2011;19:17852-63. 
27. Arun S, Choudhury V, Balaswamy V, Supradeepa VR. Octave-spanning, continuous-wave supercontinuum generation with record power using standard telecom fibers pumped with power-combined fiber lasers. Opt Lett. 2020;45:1172-5.

28. Kobtsev S, Smirnov S. Modelling of high-power supercontinuum generation in highly nonlinear, dispersion shifted fibers at CW pump. Opt Express. 2005;13:6912-8.

29. Soh DBS, Koplow JP, Moore SW, Schroder KL, Hsu WL. The effect of dispersion on spectral broadening of incoherent continuous-wave light in optical fibers. Opt Express. 2010;18:22393-405.

30. Li Q, Zhang H, Shen X, Hao H, Gong M. Phenomenological model for spectral broadening of incoherent light in fibers via self-phase modulation and dispersion. J Opt. 2016;18:115503.

31. Churkin DV, Kolokolov IV, Podivilov EV, Vatnik ID, Nikulin MA, Vergeles SS, et al. Wave kinetics of random fibre lasers. Nat Commun. 2015;6:6214.

32. Turitsyn SK, Bednyakova AE, Fedoruk MP, Papernyi SB, Clements WRL. Inverse four-wave mixing and self-parametric amplification in optical fibre. Nat Photonics. 2015;9:608-14.

33. Goodman JW. Statistical Optics. 2nd edition. John Wiley \& Sons, Inc; 2015.

34. Kelleher EJR, Travers JC, Popov SV, Taylor JR. Role of pump coherence in the evolution of continuous-wave supercontinuum generation initiated by modulation instability. J Opt Soc Am B. 2012;29:502-11.

35. Castelló-Lurbe D, Vermeulen N, Silvestre E. Towards an analytical framework for tailoring supercontinuum generation. Opt Express. 2016;24:26629-45.

36. Pinault SC, Potasek MJ. Frequency broadening by self-phase modulation in optical fibers. J Opt Soc Am B. 1985;2:1318-9.

37. Kelleher EJR. Pump wave coherence, modulation instability and their effect on continuous-wave supercontinua. Opt Fiber Technol. 2012;18:268-82.

38. Ye J, Xu J, Zhang Y, Song J, Leng J, Zhou P. Spectrum-manipulable hundred-watt-level high-power superfluorescent fiber source. J Lightwave Technol. 2019;37:3113-8.

39. Ropp C, Bachelard N, Barth D, Wang Y, Zhang X. Dissipative self-organization in optical space. Nat Photonics. 2018;12: $739-43$.

40. de Araujo MT, Da Cruz HR, Gouveia-Neto AS. Self-phase modulation of incoherent pulses in single-mode optical fibers. $J$ Opt Soc Am B. 1991;8:2094-6.

41. Churkin DV, Gorbunov OA, Smirnov SV. Extreme value statistics in Raman fiber lasers. Opt Lett. 2011;36:3617-9.

42. An Y, Huang L, Li J, Leng J, Yang L, Zhou P. Learning to decompose the modes in few-mode fibers with deep convolutional neural network. Opt Express. 2019;27:10127-37.

43. Turitsyn SK, Bednyakova AE, Fedoruk MP, Latkin Al, Fotiadi AA, Kurkov AS, et al. Modeling of CW Yb-doped fiber lasers with highly nonlinear cavity dynamics. Opt Express. 2011;19:8394-405.

44. Travers JC, Popov SV, Taylor JR. A new model for CW supercontinuum generation. In: 2008 Conference on Lasers and Electro-Optics and 2008 Conference on Quantum Electronics and Laser Science. 2008;paper CMT3. https://doi.org/10.11 09/CLEO.2008.4551286.

45. Churkin DV, Smirnov SV, Podivilov EV. Statistical properties of partially coherent Cw fiber lasers. Opt Lett. 2010;35: 3288-90.

46. Randoux S, Dalloz N, Suret P. Intracavity changes in the field statistics of Raman fiber lasers. Opt Lett. 2011;36:790-2.

47. Suret P, Koussaifi RE, Tikan A, Evain C, Randoux S, Szwaj C, et al. Single-shot observation of optical rogue waves in integrable turbulence using time microscopy. Nat Commun. 2016;7:13136.

48. Vanholsbeeck F, Martin-Lopez S, Gonzalez-Herraez M, Coen S. The role of pump incoherence in continuous-wave supercontinuum generation. Opt Express. 2005;13:6615-25.

49. Picozzi A, Garnier J, Hansson T, Suret P, Randoux S, Millot G, et al. Optical wave turbulence: Towards a unified nonequilibrium thermodynamic formulation of statistical nonlinear optics. Phys Rep. 2014;542:1-132.

\section{Publisher's Note}

Springer Nature remains neutral with regard to jurisdictional claims in published maps and institutional affiliations.

\section{Submit your manuscript to a SpringerOpen ${ }^{\circ}$ journal and benefit from:}

- Convenient online submission

- Rigorous peer review

- Open access: articles freely available online

- High visibility within the field

- Retaining the copyright to your article

Submit your next manuscript at $\boldsymbol{\Delta}$ springeropen.com 